\title{
BALADE DANS LES RUELLES «PACEÑAS» SUR LES TRACES DE L'ART MURAL URBAIN. REPRÉSENTATIONS ARTISTIQUES DES ÉPISTÉMOLOGIES AUTOCHTONES, DES DisCOURS INDIGÉNISTES ET POLITIQUES EN BOLIVIE
}

Jordie Blanc Ansari

Université Sorbonne Nouvelle Paris 3, France ${ }^{1}$

\begin{abstract}
Cet article suit les pas d'une balade dans les ruelles de La Paz, capitale administrative de la Bolivie. Nous découvrons pas à pas d'immenses fresques murales qui témoignent tout à la fois des luttes sociales et indigénistes, de l'histoire des héros mythiques et des savoirs autochtones. Au fil des rues, d'autres images apparaissent, celles de la propagande électorale, celles des contestations du pouvoir établi. Nous cherchons à comprendre les enjeux de cet art mural urbain. Nous nous interrogeons sur la fonction, les dimensions symboliques et narratives de ces graffitis afin de replacer ces pratiques artistiques collectives dans le processus historique, social et politique de la Bolivie contemporaine.

Mots clés: peintures murales, graffitis, Bolivie, Pachamama, indigène
\end{abstract}

\section{INTRODUCTION}

Je déambule dans les ruelles de La Paz, capitale administrative de l'État Plurinational de Bolivie. Je suis secouée à droite et à gauche dans le mini-bus, tout comme les petits grigris de bénédiction de la vierge de Copacabana (lieu de rituel pour les véhicules). Ici, pas d'abri-bus, peu de stations d'arrêt, pas de ticket. Le chauffeur s'arrête dès qu'il est interpellé ou qu'un passager souhaite descendre. "Aqui me quedo!» ${ }^{2}$ hurle une jeune fille en talon qui souhaite descendre devant la porte d'entrée de sa banque. Arrêt ciblé, pas une rue plus loin, pas une rue avant au risque d'entendre de longues jérémiades. Quatorze passagers peuvent se joindre au tintamarre

1 Centre de Recherche et de Documentation sur les Amériques. Jordie Blanc Ansari est doctorante en Anthropologie à l'Institut des Hautes Etudes de l'Amérique Latine - Centre de Recherche et de Documentation sur les Amériques (CREDA), Université Sorbonne Nouvelles Paris 3. Jordie travaille particulièrement sur l'appropriation sociale et politique du concept du Vivir Bien en Bolivie. Elle utilise une méthode participative par l'image auprès des acteurs locaux. Sa thèse est dirigée par Franck Poupeau. Contact: jordie.ansari@gmail.com. 2 «Je m'arrête ici!» 
des klaxons et aux freinages intempestifs. Ici, toutes les catégories sociales se mêlent: les cholitas, femmes d'origine aymara en vêtements traditionnels (grands jupons superposés, deux tresses se rejoignant par des pompons et chapeau melon), les hommes et femmes d'affaires, les étudiants, quelques touristes, les familles avec leurs enfants qui restent debout par manque de place, les festifs à l'odeur âcre d'ivresse dont le visage vacille par somnolence, les jeunes assis à l'avant avec leurs casques sur les oreilles font office d'assistants en ouvrant et refermant la porte coulissante, les jeunes filles bavardant au téléphone ou encore les intellectuels assis au fond avec un bouquin de sociologie. Dans ce spectacle assourdissant de cris, de musiques, de klaxons, de voix, de discussions, de pleurs de bébé, le regard se perd de l'autre côté des vitres. Tandis que l'attention de chacun est captée par des divers intérêts, rivée sur les performances des circassiens argentins ou sur le triste regard des marchandes de Potosi assises au pied des feux tricolores, je me concentre à observer les mûrs. À chaque intersection, je découvre une peinture murale, un graffiti, un slogan au message politique ou poétique. Ces images comblent le vide du regard, ils dissimulent les tâches, les trous, les imperfections de ces bâtiments en brique ou de ces mûrs lissés à la chaux. Je suis captivée par les détails imagés faisant référence à des bribes de l'histoire indigène, aux savoirs et aux cosmogonies des peuples autochtones ou encore aux discours politiques du gouvernement d'Evo Morales. Quels sont les enjeux de ces arts muraux? Est-ce une manière de valoriser les épistémologies des acteurs longtemps laissés en dehors de la sphère décisionnelle? Est-ce un processus visant à renforcer l'organe exécutif à travers la promulgation de son discours politique dans la rue? Ou tout simplement assistons-nous à la "prise de pinceau» pour témoigner de la réalité sociale et symbolique de ce temps là? Autrement dit, inclure l'art dans la dynamique de la vie socio-culturelle. Après avoir sillonné la avenida 20 de octubre, je m'arrête au coin de la calle Guachalla et je poursuis mon chemin dans l'étroit passage Medinaceli. Je retrouve une peinture que j'avais repérée quelques jours plus tôt. Je sors mon appareil photo et je me lance alors dans une chasse à l'image le long des ruelles, des bordures de routes et des places de la ville. En capturant ces couleurs vives, je m’interroge sur leurs fonctions, leurs dimensions symboliques et narratives. Je cherche à replacer cette pratique artistique collective et individuelle dans le processus historique, social et politique de la Bolivie contemporaine.

L'objectif de cet article est de présenter et d'analyser ces différentes images de rue en mettant en perspective ces clichés face aux enjeux politiques et aux symboles auxquels ils renvoient. Les extraits de carnet de terrain seront à la première personne afin de plonger le lecteur dans le regard du chercheur cheminant dans les ruelles paceñas. L'emploi du «nous» sera utilisé pour l'analyse. Nous étudierons cet art mural à travers trois axes. Le premier s'intéressera à la mise en récit des luttes autochtones et du discours indigéniste. Le second traitera des épistémologies autochtones. Enfin le troisième s'orientera vers l'usage politique de ces images à la fois comme outil de propagande politique et de contre-pouvoir. Nous analyserons notamment la communication visuelle du président Evo Morales omniprésente dans les rues de La Paz et E1 Alto. Cette analyse des peintures murales risque de sur-interpréter les messages des artistes. Cependant, elle cherche à donner du sens aux contenus et aux formes dessinées à partir d'une lecture anthropologique. 


\section{MISE EN RÉCIT DES LUTTES AUTOCHTONES ET DU DISCOURS INDIGÉNISTE}

\section{Luttes autochtones et mouvements sociaux}

Dans l'angle du passage Medinaceli, je découvre d'immenses peintures murales. Les garages délabrés et les poubelles débordantes contrastent avec l'esthétisme. Ces œuvres ont été conçues dans le cadre des rencontres d'arts muraux latino-américains, où des artistes de plusieurs pays sont venus colorer les rues paceñas. Cette initiative est financée par l'État. Chaque année, elle porte sur des thématiques chères au gouvernement telles que «les voix de la liberté» (2016) ou encore «les racines latino-américaines» (2018). Ce travail collectif rend hommage aux voix des acteurs subalternes, notamment les femmes et les peuples autochtones qui ont longtemps été mis de côté par l'oligarchie bolivienne. Le caractère collectif émane du défi artistique lancé par ces rencontres. En mêlant les histoires singulières des différents peuples autochtones du continent, l'enjeu est de créer une «œuvre» urbaine collective. Ce sont les thématiques qui rassemblent ces subjectivités. Certaines peintures ont été réalisées par un groupe, d'autres par un seul artiste. Dans tous les cas, c'est l'ensemble de ces pratiques artistiques qui forment le collectif.

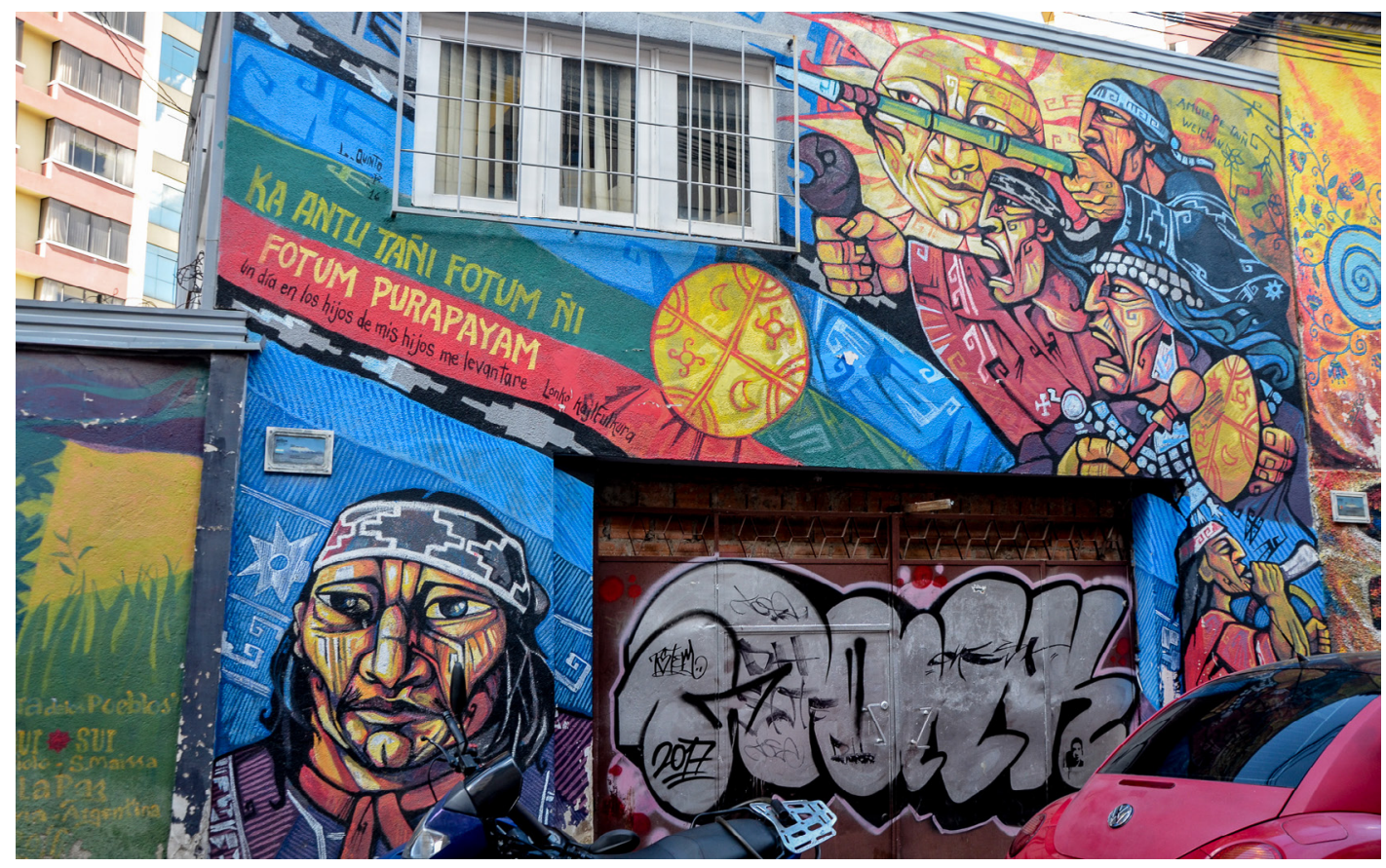

Photographie $1 .^{3}$

Je m'arrête devant une peinture murale qui représente le peuple Mapuche en lutte (voir photographie 1). Cette fresque est l'œuvre de l'Argentin Lucas Rafael Quinto et a été réalisée dans le cadre de ces rencontres Latino-américaines en 2018. Elle s’intitule: "Hommage à Calfucura Lonko Mapuche". Calfucura fut un cacique mapuche du XIXe siècle qui a défendu ardemment la reconnaissance et les droits du peuple mapuche en Argentine. Originaire de la Patagonie orientale, il voyagea dans les régions voisines afin d'apaiser les tensions et les guerres inter-ethniques. Sur la fresque, Calfucura est entouré de bleu, référence à son nom qui signifie 
«Pierre bleue». En bas à droite, un personnage souffle dans une corne comme pour appeler les autres à se joindre à la lutte. Au dessus de lui, un groupe de guerriers avancent, armé de lances et d'autres armes traditionnelles. Il lance un cri de guerre sous la bénédiction du soleil. Au centre, nous observons le drapeau mapuche Wallmapu. Ce drapeau a émergé dans les années 1990, lorsque les luttes autochtones ont pris un tournant global. A cette époque, les organisations indigènes des pays voisins se sont rapprochées pour reconstruire une histoire commune et unir leur lutte. Cette analyse peut se mettre en perspective avec l'appellation Abya Yala. Abya Yala représente le continent latino-américain. Il s'agit d'un terme emprunté à la langue Kuna du Panama et signifie «terre dans sa pleine maturité». Dans les langues natives boliviennes, ce terme pour définir le continent n'existe pas, comme le précise l'historien bolivien Pablo Quisbert. Ce sont les mouvements indigènes des années 1980, 1990 qui ont fait émerger ce terme pour renforcer leurs discours décoloniaux. Cette construction sert de catégorie pour dialoguer à l'échelle régionale mais devient également un outil de lutte politique. L'image de la lutte mapuche et de son héros mythique n'est pas sans rappeler les luttes des autres peuples autochtones de la région, notamment en Bolivie où le caractère indigène est rentré dans les discours politiques.

Cette peinture murale me fait penser à une autre fresque aperçue à El Alto. Cet ancien quartier défavorisé et périphérique de $\mathrm{La} \mathrm{Paz}$ est aujourd'hui une ville légale qui compte plus d'un million d'habitants. Cette ville populaire est composée majoritairement d'aymara venus des mines ou des terres de l'Altiplano dès la seconde moitié du XXe siècle. En contrebas d'un poteau de téléphérique, le long de la ligne bleue, une image raconte l'histoire de la lutte indigène bolivienne. Je reconnais notamment le héros Tupak Katari, accompagné de son épouse Bartolina Sisa, mais aussi Evo Morales qui se mêle à la révolte. Cet œuvre du collectif Mano Negra retrace les luttes indigénistes qui ont parcouru la fin du XVIIIe et le début du XXIe siècle. Tupak Katari avait mené un soulèvement contre la Couronne en 1781 à La Paz (Lema in Labrousse 2003). Ecartelé, par les Espagnols, il aurait annoncé à ses bourreaux, juste avant sa mise à mort: «Vous ne faites que me tuer, un jour je reviendrai, et nous serons des millions.» Cette citation célèbre a été reprise par Evo Morales en 2005 en insistant ainsi sur sa descendance indigène (Do Alto, Stefanoni 2008). Tupak Katari est devenu une figure symbolique en Bolivie. Dès les années 1970, avec l'arrivée de quelques indigènes au sein de postes de pouvoirs, des réformes apparaissent en faveur des peuples autochtones. L'université s'ouvre peu à peu aux individus d'origine indigène. Émerge alors le mouvement katariste, nom inspiré du martyr Tupak Katari, cité précédemment. Ce mouvement est porté principalement par des intellectuels ou étudiants d'origine indigène. Le mouvement katarisme reprend la légende de l'Incari, qui annonce le retour de l'Inca dans la sphère politique (Favre 1996; Do Alto et Stefanoni 2008). Ce mouvement cherche à rendre compte de la diversité culturelle et à valoriser les traditions autochtones. Ces intellectuels aymara puisent dans la mémoire collective pour repenser la question de l'identité, de l'État et de la Nation. Au cours des années 1990, dans toute l'Amérique latine résonnent des marches et des mobilisations pour défendre les droits des peuples autochtones et revendiquer leur autodétermination.

L'image du Président Evo Morales sur cette peinture fait également référence à son investiture en 2006, qui avant même son entrée au parlement bolivien, avait reçu la bénédiction des autorités religieuses aymara sur le site archéologique de Tiwanaku (Canessa 2012). Cet événement "traditionnel" marque la volonté de ce président d'asseoir son "indianité" et son alliance avec le bloc indigène. Par cet acte, il reconstitue un nouveau lieu de mémoire, Tiwanaku devient un symbole de la culture et de l'identité bolivienne. Le gouvernement d'Evo Morales 
s'inscrit dans la continuité de cette histoire. La présence de nombreuses personnalités politiques au cours des cérémonies, met en exergue une proximité à la fois entre le politique et le religieux et entre la politique et le peuple. Le fait d'assister et de participer à la fête, crée ces liens, qui tendent vers la reconstitution du pouvoir de «l'indien». Ces peintures retracent ainsi l'histoire des mouvements sociaux et indigénistes et restituent l'État dans la filiation de ces luttes de l'époque coloniale et de la période contemporaine. Ce discours politique réinvente son histoire.

\section{Réécriture de l'histoire et décolonialisme}

Au bout du passage Medinaceli, juste avant d'arriver à l'enseigne de l'hipermaxi se dévoile une fresque historique créée dans le cadre de la deuxième rencontre latino-américaine d'art mural en 2016 sur la thématique "les voix de la liberté" (voir photographie 2). La peinture est réalisée par un collectif d'artiste chiliens: Luis Pasten Tapia, Patricio Madera Mac Kicrroy, Robinson Avello Ayala et Carlos Villamar Tulcanaza. La fresque s’intitule «Résistance à l'envahisseur». Une fois de plus, la référence à l'histoire met en lumière les acteurs indigènes. La première partie à gauche représente le monde avant l'arrivée des espagnols. Un monde sublimé où la Nature vit en harmonie avec les humains. Les éléments symboliques des sociétés pré-hispaniques sont illustrés: soleil (dieu Inti), les montagnes enneigées et les forêts. Puis apparaît Christophe Colomb qui marque un tournant historique et qui regarde vers la droite. Au centre le personnage de Simon Bolivar apparaît de nouveau rappelant l'indépendance face à la Couronne espagnole. Enfin nous percevons les syndicats miniers et les peuples indigènes en lutte des années 2000 qui avancent et se confrontent à ce modèle impérialiste. Ils font face à Christophe Colomb. Il est intéressant de noter que la direction du regard des personnages renvoie aux différentes visions du temps, celle de l'occident et celle des indigènes. L'équipage de Christophe Colomb regarde vers la droite, vers le futur et les mouvements sociaux syndicalistes et les groupes indigènes regardent vers la gauche. Selon le principe andin, nous avançons vers le futur en regardant le passé, le dos tourné, car le passé se trouve devant nous et le futur derrière nous. Cette posture décoloniale réinvente le passé pour définir l'idéal de demain: un monde qui prendrait sa revanche sur la conquête espagnole.

La fresque retrace ainsi l'histoire de la Bolivie, et plus largement de l'Amérique Latine à partir d'un point de vue non euro-centré. Elle rappelle la construction du bloc social indigène-paysan durant les événements des années 2000. D'abord la guerre de l'eau en 2000 qui éclate à Cochabamba (Crespo 2000) puis la guerre du gaz en 2003 qui surgit à El Alto, au nord de La Paz. Un climat social et politique brûlant, qui entraînera en octobre 2003 la démission de Gonzalo Sanchez de Lozada puis de son remplaçant Carlos Mesa en 2005. À travers le parti politique du MAS (Movimiento Al Socialismo), Evo Morales, leader cocaleros d'origine aymara, arrive à la présidence du pays le 5 décembre 2005. Pour la première fois, la Bolivie voit un président indigène à son gouvernement, bien que cette identité soit fortement contestée. Pour les militants du MAS, parti politique d'Evo Morales, la république est l'héritière directe de la conquête. Ils perçoivent l'État-nation comme un vecteur d'assimilation des peuples indigènes. Leur projet politique intitulé «Bolivia cambia, Evo cumple» marquera une véritable rupture à travers la proclamation d'un État Plurinational et la création d'une nouvelle constitution reconnaissant les diversités culturelles des sociétés indigènes. Dès le préambule, il est question de rompre avec la république et le néolibéralisme, les peuples autochtones sont valorisés et notamment les cultures aymaras et quechuas. 


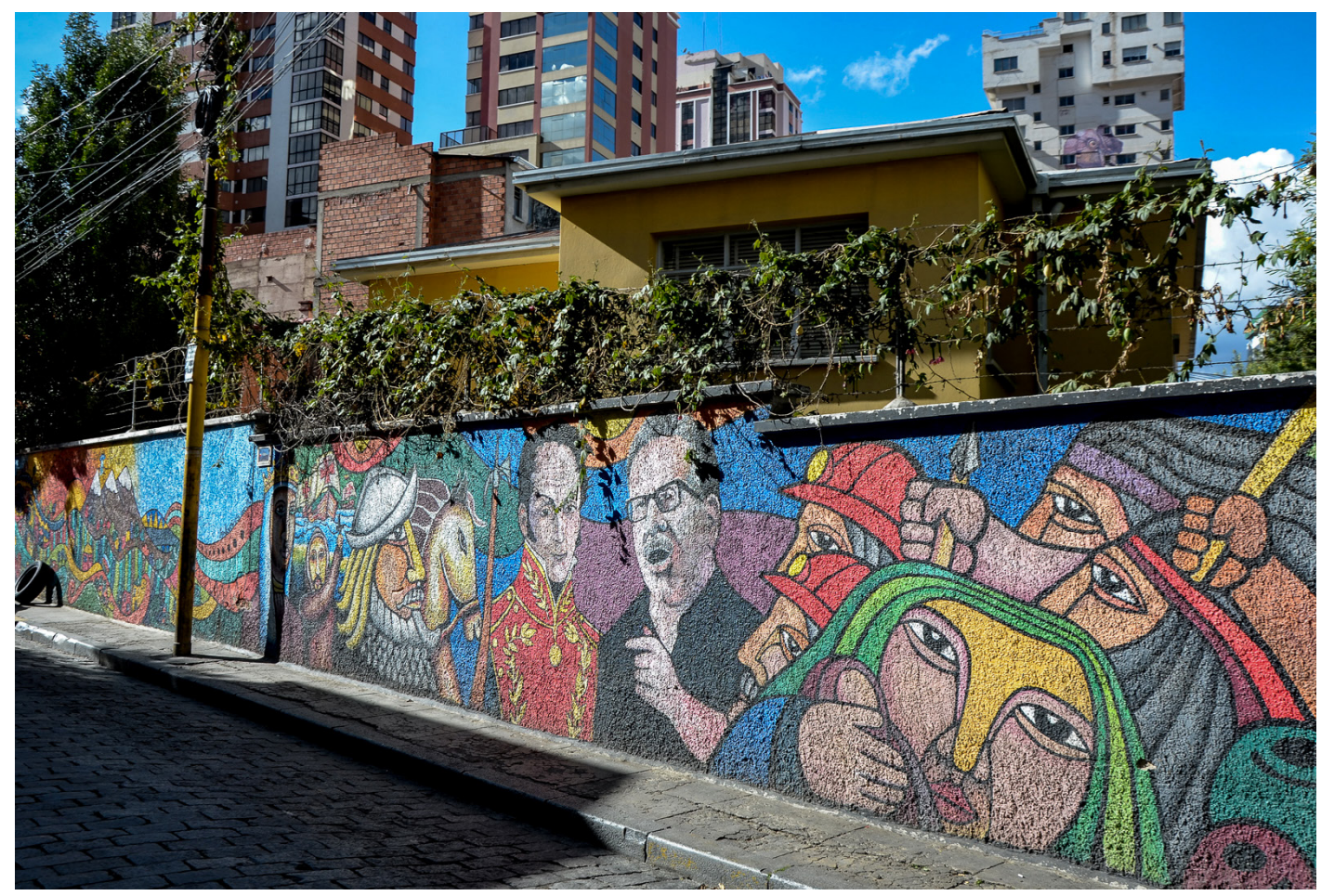

Photographie 2.

En poursuivant mon chemin, une autre image renforce cette volonté politique. Limage représente une alliance entre d'une part l'État bolivien (drapeau sur la main gauche) et d'autre part l'État vénézuelien (drapeau sur la main droite) (voir photographie 3). Ces mains reposent sur le drapeau multicolore en damier, emblématique des peuples indigènes de la Cordillère des Andes, la Wiphala. Cela marque l'alliance entre les deux États dans leur histoire et leur lutte commune pour l'indépendance et le décolonialisme. Le premier portrait situé à gauche représente Bartolina Sisa (combattante aymara, cheffe politique et militaire du XVIIIe siècle) et le personnage lui faisant face, à droite, représente Tupac katari, son époux, rebelle et leader indigène du XVIIe siècle. Bartolina Sisa est aujourd'hui le nom de l'organisation de femmes indigènes en Bolivie. Ces deux héros indigènes situés de part et d'autre semblent à la fois être le pilier sur-lequel repose l'histoire de la revendication mais peut aussi se lire comme une frise chronologique qui rappelle l'idée du Pachakuti, du «retour de l'indien» dans la sphère politique. Au centre nous observons Simon Bolivar, héros de l'indépendance de l'Amérique du Sud qui donnera son nom à la Bolivie, et Hugo Chavez, ancien président socialiste du Vénézuela et initiateur d'une nouvelle gauche en Amérique Latine. En arrière plan, nous percevons un soleil qui rayonne au delà du cadre et qui souhaite s'étendre au delà des frontière établies par l'État/ Nation. Cette allégorie de lutte contre le colonialisme et le modèle hégémonique semble reposée sur les héros indigènes et tournée vers l'avenir grâce au partenariat avec le Venezuela ${ }^{4}$. Rappelons que ce dernier a participé au financement de la première campagne d'Evo Morales. Le discours s'inscrit dans la filiation à la fois des leaders indigènes et des politiques progressistes sud-américaines. Notons que ces mêmes portraits sont également affichés dans les salles de classes. En effet, selon les directives de la nouvelle Constitution de 2009, l'éducation a pour mission de «décolonialiser» la pensée des citoyens. ${ }^{5}$ Dans le cadre d'un terrain ethnographique

4 Cette vision est à replacer dans le contexte des premières années de la présidence d'Evo Morales. Elle reste néanmoins un symbole de «la gauche» en Amérique Latine du début du XXIe.

5 Les nouveaux programmes tentent d'enseigner aux élèves l'histoire des politiques de gauches mais également l'histoire des acteurs subalternes, notamment des femmes, des peuples autochtones, des minorités (Astorga 
dans un village en Amazonie bolivienne, j'ai pu observer dans la classe des sciences sociales des affiches de Simon Bolivar, du Che Guevara et de Fidel Castro. Des élèves ont construit et suspendu au plafond une mappe-monde encerclée par un aigle géant. C'est-là le symbole de l'impérialisme nord-américain, "prédateur et dominateur du monde», comme il est souvent décrit dans les discours politiques. Cette réécriture de l'histoire est une manière de sortir du modèle néolibéral et de fonder un récit pluri-national à partir d'autres principes et d'autres héros.

A gauche de la peinture murale présentant l'alliance entre les rebelles indigènes, la Bolivie et le Venezuela (image 3), est annotée une citation de Simon Bolivar. Cette-dernière insiste sur le caractère commun prédestiné de la rébellion: «L'unité de notre peuple n'est pas une simple chimère des hommes, mais le décret inexorable du destin.» Cette fresque a été réalisée en 2017 par le collectif d'artistes «Sajra» composé de jeunes de la ville de El Alto. Cet œuvre semble un appel à la mémoire collective portant sur la liberté et la lutte. Ajoutons, que les couleurs et les références visuelles de cette fresque murale rappellent les peintures de l'artiste aymara Mamani Mamani présentes dans La Casa del Pueblo. ${ }^{6}$ Les symboles illustrés proviennent de la cosmogonie andine. Nous observons la croix andine Chacana, le Dieu Soleil, le drapeau en damier Wiphala, la tête du puma, du serpent et du condor, autrement dit les animaux emblématiques de la culture andine. Sur la porte centrale le portrait des héros indigènes est gravé, dominé par le blason du drapeau national. Une façon symbolique de raconter les ambitions politiques du gouvernement, soit de conduire le peuple vers un État Plurinational avec un paradigme de développement fondé sur les cosmogonies autochtones tout en restant dans le cadre de l'Étatnation. Cette ambiguïté reste néanmoins au cœur des débats de cette politique située entre deux mondes: d'une part «la matrice ancestrale millénaire et (d'autre part), la matrice occidentale centenaire, qui est arrivée en 1522 avec l'invasion espagnole et la mort d'Atahualpa» (Yampara 2010).

Je longe l'Avenida 6 de Agosto à contresens, interpellée par les vendeurs ambulants. Je m’interroge sur les multiples dimensions de ce discours décolonial. Je traverse la grande place faisant face à l'université publique de La Paz, la UMSA. A travers les grilles, je découvre d'autres fresques qui recouvrent l'enceinte du bâtiment. La figure du Che Guevara marque nettement la position politique de rompre avec le modèle capitaliste et du tournant vers une «nouvelle gauche» qui a émergé en Amérique Latine à partir des années 2000 et jusqu'en 2019, si nous concluons cette période par la démission d'Evo Morales. Mais l'indianisme est-il de gauche? Interroge Hervé Do Alto et Franck Poupeau dans un article publié dans la revue Civilisation en 2009. Une citation accompagne la fresque du Che Guevara: «Toute notre action est un cri de guerre contre l'impérialisme». La question de la décolonialité recouvre ainsi deux enjeux essentiels. Tout d'abord concernant la restitution des droits des peuples originaires en valorisant leurs pratiques traditionnelles, leurs croyances, leurs cosmologies afin de les considérer comme des citoyens égaux. Ensuite, il s'agit de contrôler le «pillage» des richesses nationales pas les compagnies étrangères. En somme, le cœur du débat sur la décolonialité tourne autour de la souveraineté nationale sur les ressources naturelles et l'autodétermination des peuples. Ces deux éléments sont de fait, parfois contradictoires car un même territoire est parfois animé par un double intérêt. Or, la notion même de territoire est à déconstruire car elle délimite l'espace selon une perspective politique, tandis que les sociétés andines considèrent l'espace et la société autour d'un même principe symbolique, dans le sens où les deux sont en interconnexion et in-

2012; Lewandowski 2015; Minteguiaga 2012).

6 Cet édifice a été construit de 2014 à 2018 et loge plusieurs ministères ainsi que le gouvernement. 
terdépendance. Ce discours politique qui cherche à rompre avec le modèle hégémonique prône un autre paradigme de vie fondé sur le Vivir Bien.

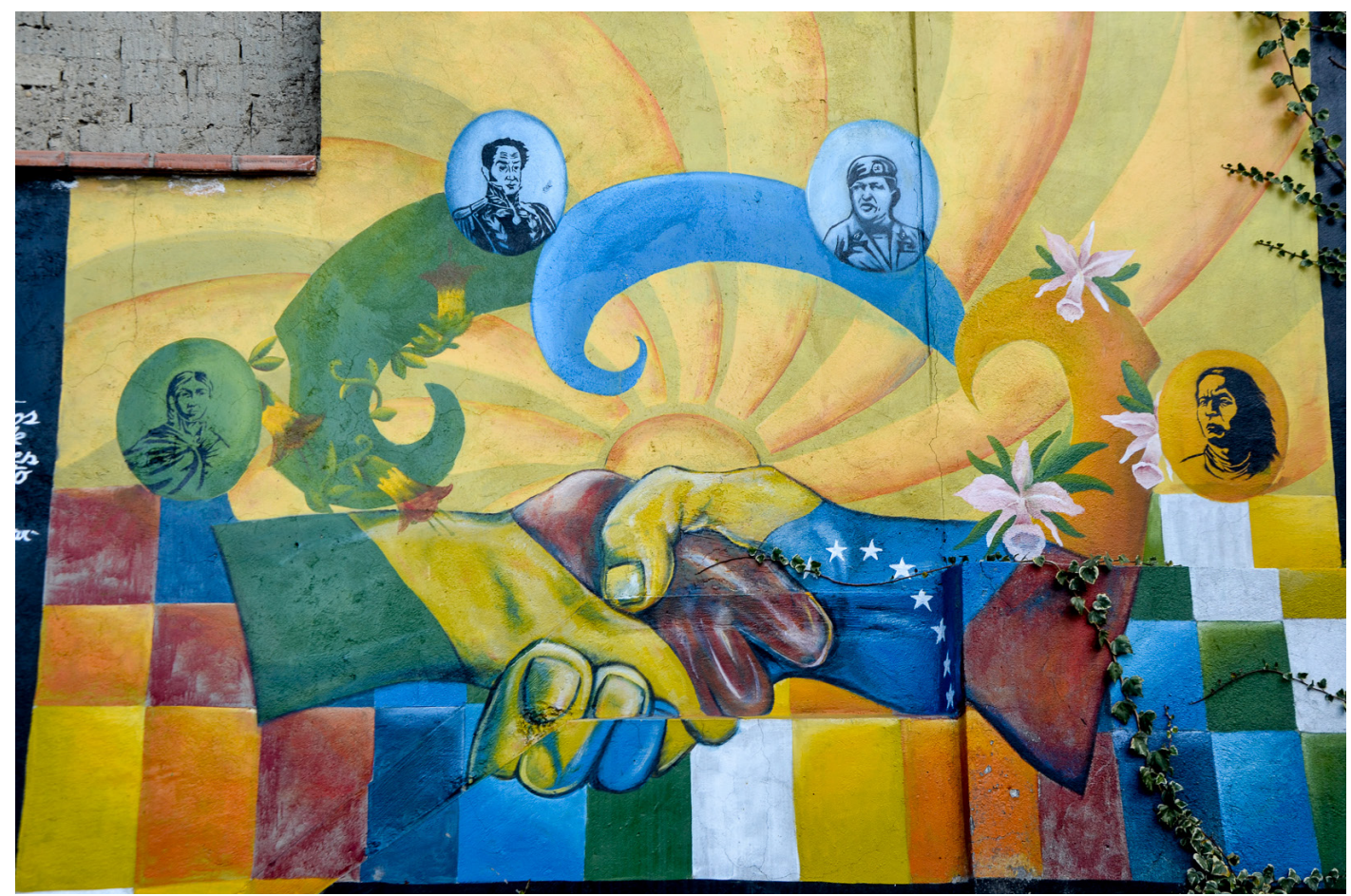

Photographie 3.

\section{ÉPISTÉMOLOGIES AUTOCHTONES: LA REPRÉSENTATION DE LA PACHAMAMA ET DE LA VISION BIOCENTRIQUE}

Je poursuis mon chemin en direction de la Camacho, immense marché au cœur de la ville et départ du téléphérique Céleste. Au bout de quelques minutes de marche, je me faufile dans une cabine. Je m'envole au dessus de La Paz, «ciudad del cielo ${ }^{7}$ » comme le souligne la municipalité sur les affiches publicitaires. Je survole le marché et j'admire les immenses peintures murales à travers la vitre. Ces décors colorés montent jusqu'en haut de la colline à l'espace de jeu Pipiripi. Cette fois, les symboles renvoient directement à la cosmogonie andine. Une des plus grande peinture réalisée lors de la première édition des rencontres d'arts muraux représente une femme qui lève le menton et porte des montagnes enneigées dans ses bras. Entourée de rivière et de forêt elle signifie l'harmonie entre tous les êtres vivants. La féminité renvoie à la Pachamama, la Terre-Mère mais aussi à la vision biocentrique. En effet d'après la cosmogonie andine, tous les êtres vivent en interconnexion et en interrelation.

La proposition du Vivir Bien propose un autre regard sur l'altérité. Le concept est traduit du kichwa, Sumak Kawsai, et de l'aymara, Suma Qamaña. Selon cette conception, la Nature acquiert une place toute aussi importante que l'homme, où le «bien vivre» s'apparente à l'harmonie qu'entretiennent les êtres vivants les uns avec les autres. Nous l'entendons officiellement pour la première fois en 2008 lors de son inscription dans la Constitution équatorienne, le

$7 \quad$ Traduction de l'auteur: «Ville du ciel» 
premier État à accorder un droit à la Nature, puis en 2009 dans la nouvelle Constitution bolivienne. La Nature est une catégorie plurielle et désarticulée qui ne représente pas un tout unifié. Elle méle tous les éléments de l'ordre du vivant. Dans les représentations symboliques des populations andines, le vivant est considéré comme un des facteurs essentiels à l'épanouissement de la société. Le Vivir Bien s'apparente ainsi à une véritable philosophie qui installe tous les étres sous un méme pied d'égalité même si chacun est différent de part son espèce. La Nature a jusque là était considérée par les politiques environnementales et les acteurs du développement comme un outil à la disposition de l'homme. En ce sens, les actions humaines se sont appropriées ces différents éléments dans le but d'augmenter leur capital économique, leur nombre d'exportation et de mettre en place des relations commerciales (Gudynas 2014). La Nature est devenue tour à tour la propriété d'un État, d'une entreprise, d'un privé ou d'une collectivité. Ce sont ainsi multipliée les exploitations massives agricoles, forestières et minières, de façon à assouvir les besoins économiques et politiques sans prendre en considération les conséquences environnementales de ces actions. Précisons que ces dernières ont des répercutions directes sur la société puisque l'homme et la nature sont en interaction continue. Ces activités cherchent avant tout à développer l'économie des États sans accorder à l'environnement une valeur en soi. Les mouvements dits écologistes chargés de «défendre l'environnement» mettent en avant l'utilité de la protection de la nature, à la fois pour la production d'oxygène mais aussi comme patrimoine à laisser en héritage aux générations futures. Or, ces mouvements sont eux-aussi tournés vers les besoins de l'homme. L'anthropocentrisme impose de cette façon a l'objet, des valeurs qui lui sont extérieures. «Une plante, un animal, une cascade, ne présente pas de valeur en soi, c'est l'être humain qui lui en attribut pour lui-même» (Ibid). La nature est donc marquée par son utilité. Prenons l'exemple d'un arbre devenu une pièce de bois, sa valeur réside dans l'utilisation de son bois. L'arbre en tant qu'être vivant ne possède pas de droit ni de valeur indépendante pour lui- méme. Tandis que «le biocentrisme ${ }^{8}$ défend l'obligation morale envers les plantes et les animaux sauvages en tant que membres d'une même communauté biotique9» (Taylor 1981 in Gudynas 2014).

Les fresques de la Camacho renforcent cette vision d'interrelation entre les êtres vivants. Elles mettent sur un même support mural des animaux, des végétaux, des ressources naturelles: rivières, forêts, montagnes. Ces éléments s'entremêlent les uns aux autres comme faisant partis d'une seule unité. En réalité, le Vivir Bien met en lumière la relation biotique des deux corps, «Nature et Humanité», souvent présentés comme distincts selon l'opposition bien courante en occident, «Nature / Culture», quand bien même l'une se fond dans l'autre. Cette vision marque à la fois notre façon d'agir sur le monde mais aussi notre manière de le penser. Le Vivir Bien est une production culturelle à la fois empreinte d'une dimension affective et d'une dimension pratique. Il se revendique à la fois d'un héritage indigène et prend place dans la politique contemporaine. En Bolivie une institution gouvernementale, la Autoridad Madre Tierra, est chargée de contrôler les droits de la Pachamama. Cependant, elle ne dispose pas de jurisprudence propre malgré la loi no 071 du 21 décembre 2010, qui donne des droits à la Pachamama. La loi définit la Terre-Mère comme un: «système vivant dynamique, structuré par la communauté indivisible de tous les systèmes et êtres vivants interconnectés, interdépendants et complémentaires, qui partagent un destin commun.» ${ }^{10}$ Cette nouvelle place juridique transforme la nature en un véritable sujet de droit (Landivar, Ramillien 2013). Cependant, c'est en octobre 2012, qu'émerge

8 Le biocentrisme prend pour cadre de référence tout l'univers du vivant.

9 La biotique représente l'ensemble des interactions du vivant sur le vivant dans un écosystème.

10 Article 3 de la loi n 071 sur les droits de la Terre-Mère, 21 décembre 2010. Traduction de Victor Audubert (2017). 
une nouvelle loi offrant plus de pouvoir à l'institution. La «loi-cadre $n^{\circ} 300$ de la Terre-Mère et du développement pour Vivre Bien». Cette seconde législation n'a plus vocation d'assurer des droits à la nature mais plutôt de structurer ces droits au sein d'une autorité gouvernementale, la Autoridad Plurinational de la Madre Tierra (Audubert 2017).

Or, au delà de la représentation féminine, s'ajoute l'aspect nourricier de la Terre (Poupeau 2011) avec par exemple, le jaillissement de l'herbe ou encore le fotus que nous retrouvons sur la fresque «Esperanza de vida». Au centre de cette dernière un arbre prend sa racine et poursuit sa croissance au delà du cadre. Nous remarquons un condor qui domine le tableau, symbole de l'esprit et du cosmos dans la culture andine. Nous le retrouvons dans de nombreuses peintures murales qui rendent hommage aux savoirs andins et à la cosmogonie des peuples autochtones. Ces œuvres situées dans plusieurs quartiers de la ville amènent la culture à la portée de chaque citoyen, le long de leurs chemins quotidiens. En faisant appel aux artistes sud-américains pour colorer les rues, les acteurs politiques cherchent à embellir la ville et mettre en lumière la dimension socio-culturelle du sous-continent. Les peintures murales renvoient également à une certaine forme d'éducation. En effet, en mettant en scène publiquement ces récits historiques en marge, ces images fabriquent l'Histoire. "Cette éducation populaire doit être pensée avant tout comme une praxis culturelle de transformation sociale et politique» expose Christian Maurel dans son ouvrage Education populaire et puissance d'agir (Maurel 2010). Cet art mural urbain met en lumière les contradictions et cherche à faire conflit. Sous l'effet d'une provocation, ces pratiques artistiques nous ouvrent «les chemins d'une émancipation entendue comme dégagement de la place qui nous a été assignée par les conditions sociales, les appartenances culturelles, le genre, ou encore les handicaps de toutes sortes» (Maurel 2010). En effet, ces images combattent les formes d'ethnocentrisme social qui clivent la Bolivie (Dubois 1994 in Mensch 2016). L'enjeu de ces peintures murales est d'inscrire la figure de l'indien dans l'Histoire, non à travers l'image du vaincu, mais plutôt celle de l'indien héroïque en résistance depuis plusieurs siècle. Cette contestation inscrite sur l'espace public avec un cadre normatif, celui des rencontres latino-américaines, revêt d'un fort marqueur identitaire. Cet art mural est donc à penser comme une éducation populaire urbaine qui vise à travers la diffusion de ces savoirs, à créer du collectif.

Il n'est pas anodin que les quartiers choisis pour accueillir ces arts muraux soient la Camacho: centre ville et grand marché, la Sarganaga: quartier d'artisanat et touristique et enfin le quartier de Sopocachi. Ce projet financé par l'État remet en question le caractère subversif du street-art. Habituellement le street-art est une expression populaire de rébellion face à l'ordre établi. Or, cet engagement et encouragement politique transforme cet acte transgressif en acte culturel (Mensch 2016). Le caractère politique n'est plus celui de la contestation mais de la valorisation et de l'illustration du discours politique. En ce sens, les images de rue semblent d'avantage être les voix du haut descendues vers le bas plutôt qu'une réelle "prise de pinceau» du bas. De plus, il n'est pas anodin que le quartier de Sopocachi accueille ce projet artistique. Il s'agit d'un quartier un peu «bohème» qui a abrité de nombreux écrivains, journalistes et artistes boliviens et qui, depuis quelques années, devient de plus en plus touristique avec le développement de restaurants, de discothèques et de bars. C'est aussi un espace socio-culturel important où réside l'alliance française, des musées d'art et des librairies.

Néanmoins, l'occupation du discours politique dans l'espace public va au delà de la mise en image de l'histoire et de la cosmogonie autochtone. En effet, l'image du président est très présente dans la ville. Cela contribue à l'inscrire dans cette filiation historique mais aussi de 
lui reconnaître les projets que son gouvernement a établi ainsi qu'à encourager ses prochaines campagnes électorales.

\section{L'USAGE POLITIQUE DES IMAGES DE RUE}

\section{L'image du président Evo Morales dans la ville}

Je survole toujours La Paz dans ma cabine céleste. Suspendue dans les airs telle un funambule sur son fil, je profite du paysage. Les autres passagers restent fixés sur leur téléphone mobile, trop habitués à ce transport routinier. La cabine de téléphérique est spacieuse, avec des bancs en bois et une porte à l'ouverture automatique où l'on peut voir le logo du président Evo Morales. Ce logo représente son image entourée de sa fonction «Président de l'État Plurinational de Bolivie» de style pop-art. Nous le retrouvons sur les livres scolaires, les documents officiels, dans la ville mais aussi sur les aliments donnés aux femmes enceintes (allocations familiales). Cette image du président est aussi représentée sur d'autres supports tels que les infrastructures installées par le gouvernement ainsi que les panneaux publicitaires signalant les installations de lignes téléphériques, les réseaux de routes et les aéroports. Est-ce-un moyen d'influencer l'opinion du peuple, et tout particulièrement les citoyens illettrés pour qui le discours imagé a plus d'impact?

Comment l'État et spécifiquement le président Evo Morales occupent-ils l'espace urbain et les imaginaires? L'art visuel s’installe dans la rue d'une façon que nous pourrions qualifier d'éphémère, or, la présence dans le temps de ces images questionne ce support de rue. Le fait de marquer sa présence, est-ce une façon d'inscrire sa permanence dans le temps? Précisons qu'en octobre 2019, Evo Morales s'est présenté pour la quatrième fois aux élections présidentielles malgré le caractère anti-constitutionnel de cette candidature et le refus de la population d'élargir l'article de loi limitant le président à deux mandats consécutifs ${ }^{11}$. Finalement nous pouvons en venir à questionner le passage de l'État-Nation à l'État plurinational. Serait-on passé à un État-MAS, comme le souligne le juriste Philippe Boulanger? Étant donné que le pluralisme politique est peu encouragé et l'opposition contrôlée. Notons que depuis 2009, le parti politique du MAS représente 2/3 au parlement. Ce monopole idéologique du gouver-

11 Le 21 février 2016, les citoyens ont voté «non» au référendum concernant la modification de l'article 168 de la Constitution et qui donnait la possibilité au président de se réélire sans limite de mandat. Le gouvernement demande alors une révision de la loi au Tribunal Constitutionnel Plurinational qui fini par accepter la modification de l'article (Boulanger 2018). Lors des élections du 20 octobre 2019, Evo Morales a remporté la victoire. Des suspicions de fraude ont engendré de violentes contestations populaires. L'Organisation des États Américains s'est alors chargé de mener un diagnostic et conclu sur des traces d'irrégularités dans le comptage des votes. Au même moment, l'armée et la police ôtent leur soutien au président. Ce dernier se voit obligé de démissionner et demande l'asile politique au Mexique. Malgré l'absence de quorum, c'est l'ex-sénatrice Jeanine Añez de la droite radicale catholique, qui assure la présidence en attente de nouvelles élections. Certains dénoncent un coup d'état, d'autres une révolte démocratique (Le Monde - 18.11.2019). Dans tous les cas, après la démission d'Evo Morales, la situation reste tendue. La wiphala (drapeau indigène) qui trônait au palais du gouvernement est brûlée. Un symbole fort qui représente la plurinationalité de l'État. La nouvelle présidente prend ses fonctions avec la bible en main. Les forces de l'ordre s'en prennent aux manifestants partisans d'Evo Morales mais aussi aux citoyens d'origine indigène qui rejettent l'arrivée de la droite radicale, notamment à El Alto et à Cochabamba. Ce retour de l'oligarchie témoigne de la prégnance du racisme mais aussi de la complexité de l'opposition qui faisait face à Evo Morales (droite radicale, intellectuels de gauche, élite économique, population des basses terres, population des régions de gisements de gaz et de lithium notamment) (Le Gouil 2019). 
nement semble contradictoire aux principes même énoncés dans la Constitution qui prône le pluralisme. Finalement l'organe exécutif est renforcé avec une personnalisation du régime à travers la figure d'Evo Morales. L'ordre indigène est mythifié et participe à la légitimité du président. La rupture historique qu'il souhaite incarner cible son discours sur l'émancipation des peuples autochtones et la plurinationalité. Ce logo et les graffitis émergent de-ci de-là en faveur du président et semblent être du ressort d'une instrumentalisation politique, d'une propagande électorale. Cependant ces images de rue sont mises en perspective avec des graffitis qui contestent le pouvoir établi.

\section{Expression collective de contre-pouvoir}

Le logo du président est parfois réutilisé par les opposants selon la technique du pochoir. Ces derniers modifient le texte, à la place de «président» ils inscrivent «Dictateur de l'État Plurinational de Bolivie». «Les tags et les graff' sont des pratiques collectives. Le partage d'expériences transgressives et créatrices apporte un sentiment d'appartenance à une entité qui s'avère être, dans bien des cas, un palliatif à des mécanismes d'intégrations défaillants ou insatisfaisants» (Mensch 2014). Ces images issues de la rue visent à laisser un message politique et à soulever «les problèmes sociaux ou étiques». Olivier Dabène propose différentes hypothèses pour analyser la prépondérance de ces images dans les espaces publiques latino-américains depuis le début du XXIe (2014). Selon le politologue, il peut s'agir d'un outil de contestation utilisé par les mobilisations sociales ou encore l'expression politique d'une génération qui rejette la politique représentée. Dans tous les cas, ce phénomène nous renseigne sur la manière dont les citoyens s'emparent de l'espace public en exprimant leurs opinions politiques dans la rue. Précisons que le président Evo Morales a perdu une grande partie de son électorat. En effet, nous notons plusieurs clivages politiques. Entre d'une part l'Altiplano bolivien fervent partisan du MAS en date de 2009, et, d'autre part, l'Oriente où l'opposition est de venue majoritaire. Nous observons également un clivage entre le monde urbain et le monde rural comme le souligne Jean Pierre Lavaud, car sur les neuf capitales départementales, six se sont opposées à ce projet constitutionnel (Lavaud 2008). Enfin, il nous semble important de souligner que la nouvelle constitution valorisant la sphère indigène dérange l'oligarchie bolivienne. Cette dernière rejette totalement cette Constitution, la trouve obsolète et inefficace. Du côté des intellectuels qui ont participé à ce bouleversement idéologique, certains ont obtenu des postes au sein du gouvernement peu après sa mise en place, mais beaucoup ont été déçus. Dans de nombreux cas, la ligne politique n'est pas allée dans le sens qu'ils souhaitaient. Les intellectuels alteños contestent l'intégrité idéologique du gouvernement. Le concept «Pachamama» utilisé par la sphère politique leur semble un réservoir fourre tout, dénué de sa substance spirituelle. Ils observent de nombreuses contradictions politiques, notamment en terme d'exploitation des ressources. L'État semble encore poursuivre la logique néolibérale de profit économique. De nombreux graffitis, notamment durant la période de campagne électoral en 2019 cherchent à orienter les citoyens ou encore à prononcer ouvertement le manque de démocratie. Cependant, ces graffitis ne semblent pas être un écart à la norme quand bien même leur défi reste politique. Nous retrouvons cet usage des murs, des portes et des autres supports partout dans les villes boliviennes, les villages, le long des routes, sur les murs des maisons, des épiceries ou encore sur les panneaux officiels.

Enfin, d'autres messages muraux se glissent sur les parois. Clandestinement, ils cherchent à faire entendre leur voix sur des sujets tabous. Par exemple, le mouvement féministe «Mujeres 
creando» dénonce le machisme et attaque ouvertement le président Evo Morales. «Si Evo avait un utérus, l'avortement serait dépénalisé et nationalisé» (Dabène) Une fois de plus, le fait de s'adresser directement au président témoigne de la personnification du régime.

Ces messages sont prégnants dans les rues paceñas, parfois relevant du vandalisme, d'autres fois d'une rencontre artistique officielle, parfois allant dans le sens du gouvernement, parfois le contestant. Nicolas Mensch distingue les graffitis, des fresques peintes sur des murs qui lui sont assignées, ou parfois commandées (Mensch 2016). Alors qu'à ses prémisses, ce mode d'expression urbain se définissait par son illégalité, son caractère transgressif des normes sociales et du droit. Les peintures murales émanent quant à elles directement d'une décision politique. Néanmoins, elles s'intègrent aussi dans une démarche revendicative au vu des discours contre-hégémoniques qu'elles véhiculent. Dans tous les cas, l'émergence de l'art de rue en Bolivie devient une véritable pratique artistique à fort potentiel socio-politique. Cette expression émane à la fois d'un contexte local mais aussi d'une tendance mondiale. Le mode d'expression dans la rue n'est pas anodin, il vise à rendre public les messages véhiculés. Cette diffusion permet une transmission à un large collectif: les passants. La notion de collectif revêt ainsi une dimension plurielle car elle désigne les créateurs de ces images de rue et les passants qui les regardent.

\section{CONCLUSION}

Les murs représentent les limites de la ville, les limites entre le dedans et le dehors, entre moi et l'autre. Ces murs sont des obstacles à la vue, tel des traits verticaux reliant le ciel et la terre mais dépourvus de vie. De simples matières immobiles, stables et figées sur la Terre. Ces peintures transforment ces murs en de véritables œuvres d'art. Elles les mettent en mouvement. Elles leur donne vie. Elles bousculent les limites de notre temps et de notre imaginaire pour porter notre regard au delà de l'urbain, au delà du réel, au delà de la matière. Ce voyage artistique, historique et idéologique nous emmène en réalité au cœur des enjeux socio-politiques de la Bolivie contemporaine. En empruntant les ruelles paceñas nous découvrons ces images de rue. Ces peintures murales et ces graffitis relèvent à la fois du formel et de l'informel, tantôt encouragés par l'État tantôt visant à le controverser.

Les images urbaines sont souvent définies comme éphémères de par leur caractère temporaire et leur inscription sur des supports à priori non appropriés tels que les ponts, les bords des routes, les façades de bâtiments, les tunnels, les panneaux publicitaires. Or, les nombreuses peintures murales recouvrant les quartiers de Sopochachi, de la Camacho ou encore de la Sarganaga ont un caractère on ne peut plus permanent. En suivant pas à pas les traces de ces artistes, de cet art mural urbain, nous pouvons questionner dans quelles mesures la ville est devenue une plate-forme de communication politique. Certaines de ces images sont signées et répertoriées, d'autres sont au contraire clandestines et anonymes. À la frontière entre l'écrit et l'image, ces artistes peuplent notre culture visuelle avec des textes et des couleurs qui marquent nos esprits. La représentation des leaders aymara et quechua, des symboles des cultures autochtones tels que le drapeau indigène, la croix andine ou encore de la Terre-mère, nous emmène vers l'univers andin. Les textes quant à eux renvoient à un rejet du modèle capitaliste et de l'ingérence Etasunienne mais également à la fierté «plurinationale» des peuples boliviens. Ces nouvelles 
formes épistémiques participent à la ré-invention d'un corpus pour re-penser l'État et le «développement» à partir des savoirs autochtones.

Ces œuvres murales sont le reflet du collectif dans le sens où elles témoignent des représentations collectives des sociétés andines. C'est donc par un appel aux symboles et à la mémoire sociale que nous pouvons situer ces pratiques artistiques collectives dans une dimension sociale. De plus, les idées véhiculées par ces fresques renvoient aux luttes indigénistes et au discours politiques du gouvernement d'Evo Morales, ce qui les entraîne dans une démarche politique. Le rapport entre l'individu qu'il soit artiste, citoyen, acteur politique ou encore spectateur, et le collectif, à la fois national, pluri-national, corporatiste ou syndicaliste, semble être imbriqué autour de l'acte créatif. Le fait de placer ces peintures murales dans la rue permet d'établir un dialogue avec le passant, de l'intégrer à cette histoire collective. Le passant n'est pas seulement spectateur mais aussi passeur de cette histoire sociale et politique. Une histoire qui s'ouvre sur l'avenir en vue des événements qui secouent la Bolivie depuis la démission d'Evo Morales. Ces œuvres éphémères résisteront-elles aux tempêtes politiques? Seront-elles effacées comme l'on balaye un souvenir qui dérange ou resteront-elles intactes et les témoins des luttes et des épistémologies autochtones. Cet art mural urbain aussi divers soit-il par ses formes, ses couleurs, ses styles et ses messages est le résultat d'un travail collectif que nous pouvons inscrire dans un processus socio-politique plus large visant le renversement du modèle hégémonique occidental.

\section{BiBLIOGRAPHIE}

Astorga, Alfredo. 2012. "Sociedad educadora y sociedad del aprendizaje» in Educación y Buen Vivir: reflexiones sobre su construcción. Quito, Ecuador, edited by Otto Zambrano Mendoza.

Audubert, Victor. 2017. «La notion de Vivir Bien en Bolivie et en Equateur, réelle alternative au paradigme de la modernité?» in Cahiers des Amériques Latines $n^{\circ} 85$.

Balandier, Georges. 2013. Anthropologie politique. (1967) Paris, Quadrige.

Boulanger, Philippe. 2018. «L'Etat plurinational de Bolivie». in Problèmes d'Amérique latine $n^{\circ} 108$.

Crespo F. Carlos. 2000. «Continuidad y ruptura: la "guerra del agua" y los nuevos movimientos sociales en Bolivia» in La "guerra del agua" en Cochabamba. La cuestión agraria y el Movimiento Sin Tierra en Brasil. Buenos Aires, OSAL n ${ }^{\circ}$. Edited by CLACSO.

Dabene, Olivier, 8-11 avril 2014. «Les murs propres ne disent rien. Un nouveau muralisme dans les villes latino-américaines?» XIe Rencontre Internationale d'Études Politiques Sociales, Université de La Havane.

Descola, Philippe. 2005. Par delà nature et culture. Paris, Gallimard.

Do Alto, Hervé and Pablo Stefanoni. 2008. Nous serons des millions. Evo Morales et la gauche au pouvoir en Bolivie. Raisons d'agir, Paris.

Do Alto, Hervé and Franck Poupeau. 2009. «Lindianisme est-il de gauche? Remarques complémentaires sur l'Occident décroché». in Civilisations n58.

Favre, Henri. 1996. L'indigénisme. Paris, Presses universitaires de France.

Gudynas, Eduardo. 2014. Derechos de la naturaleza y politicas ambientales. La Paz, Plural.

Landivar, Diego and Emilie Ramillien. L'économie politique des humains et non humains: nouvelles constitutions politiques en Amérique Latine et reconfigurations ontologiques. 2013.

Lavaud, Jean-Pierre. 2008-2009. «Le vote de la Constitution bolivienne.» in Problèmes d'Amérique latine $\mathrm{n}^{\circ} 71$. 
Le Gouil, Claude. 2019. «La crise politique bolivienne vue «depuis le bas». lundimatin\#218, le 25 novembre 2019.

Lewandowski, Sophie. 2015. «Les savoirs scolaires entre mondialisation, décolonisation et hybridation. Modèles de société et éducation à l'environnement en Bolivie.» in Tiers-Monde $n^{\circ} 223$.

Maurel, Chistian. 2010. Education populaire et puissance d'agir. Les processus culturels de l'émancipation. Paris, L'Harmattan.

Mensch, Nicolas. 2016. L'art Transgressif du graffiti: pratiques et contrôle social. Doctoral thesis in Sociology, University of Franche-Comté. Under the direction of FERREOL Gilles.

Minteguiaga, Analia. 2012. «Nuevos paradigmas: educación y buen vivir» Educación y buen vivir: reflexiones sobre su construcción. Edition: Otto Zambrano Mendoza. Quito - Ecuador Nicolas, Vincent and Pablo Quisbert. 2014. Pachakuti: el retorno de la nación: estudio comprativo del imaginario de nación de la Revolución Nacional y del Estado Plurinacional. PIEB, La Paz.

Poupeau, Franck. 2011 «L'eau de la Pachamama: commentaire sur l'idée d'indigénisation de la modernité.» in L'Homme.

\title{
Strolling through the "Paceñas" Alleys on the Traces of URban MURAL ART. ARTISTIC REPRESENTATIONS OF INDIGENOUS EPISTEMOLOGIES, INDIGENOUS AND POLITICAL DISCOURSES IN BOLIVIA
}

\begin{abstract}
This article strolls through the alleys of La Paz, the administrative capital of Bolivia. We discover, step by step, the huge murals which are testimony of the social indigenous struggles, the history of mythical heroes and indigenous knowledge. As we walk through the streets, other images make their appearance, those of electoral propaganda, of challenges to the established power. We seek to understand what is at stake in this urban mural art. We question the function, the symbolic and narrative dimensions of these graffiti, in order to place these collective artistic practices in the historical, social and political context of contemporary Bolivia
\end{abstract}

Keywords: murals, graffiti, Bolivia, Pachamama, indigenous

Reçu en: 2019-08-31

Accepté en: 2019-01-20 